\title{
A Representação Social do Conhecimento: Explorando as razões para aprender e as razões para não aprender
}

\author{
Antonio Roazzi*, Hilda Bayma-Freire ${ }^{* *}$, Maira M. Roazzi ${ }^{* * *}$, e Suely Aparecida do Nascimento Mascarenhas ${ }^{* * * *}$ \\ *Universidade Federal de Pernambuco (Brasil), ${ }^{* *}$ Faculdade Anchieta do Recife, \\ ${ }^{* * *}$ Faculdade Pernambucana de Saúde, ${ }^{* * * *}$ Universidade Federal do Amazonas
}

\begin{abstract}
Resumo
Neste trabalho, exploramos uma série de hipóteses específicas com o objetivo de entender a transmissão intergeracional de status social e educacional. A perspectiva geral proposta aqui é que este fenômeno resulta de um processo de socialização que é simultaneamente cognitivo e social. Crianças de NSE baixo e médio constroem mundos consensuais diferentes que dão às crianças de NSE médio razões para aprender $\mathrm{e}$ às crianças de classe baixa razões para não aprender na escola. As diferenças que antecipamos encontrar não estão nas crenças gerais sobre o valor da educação, mas nas suas representações específicas de estratificação social e educacional, sua identidade neste mundo social e os tipos de conhecimentos nos quais se considere valga a pena investir, em função da identidade.

Palavras chave: Socialização, representação social da inteligência, psicometria, aprendizagem, autoconceito.
\end{abstract}

A análise da transmissão, de geração a geração, de status social e sua relação com a educação tem visto ondas de pesquisa e de teoria com orientações marcadamente diferentes. A evidência inicial de uma associação entre desempenho em testes de inteligência e classe social (Bayley \& Jones, 1937; Terman \& Merril, 1937) junto com a visão da sociedade como uma meritocracia (Herrnstein, 1971) permitiram que esta transmissão entre gerações ocorresse, no passado, sem problemas. Escolas podiam ser consideradas caixas pretas (French, 1990), onde crianças aprendiam de acordo com suas habilidades e sua motivação; quando deixavam a escola, a sociedade colocava-os em empregos que eram adequadas ao seu êxito educacional. A teoria e a pesquisa, então, eram direcionadas para determinar quanto das diferenças observadas em testes de inteligência eram consequência da hereditariedade e quanto resultava do meio. Com crescentes investimentos em educação compensatória nos anos 60 e o retorno alegadamente pequeno na mudança da transmissão entre gerações de status social e seu componente educacional ("A educação compensatória foi testada e falhou"; Jensen, 1969, p.2), alguns pesquisadores ficaram cientes da necessidade de uma mudança de paradigma. O papel das escolas na transmissão intergeracional de deficiência educacional (Bourdieu e Passeron, 1977) foi então reconhecida e documentada através de análises sociológicas de quem tem sucesso e quem reprova. No entanto, estas análises não contam a história inteira porque não há nenhum determinismo estrito entre status social e educacional através de gerações em sociedade democráticas (Bourdieu, 1974), e pesquisadores voltaram-se mais uma vez a análises mais detalhadas das trajetórias de indivíduos através da escola.

Nesta apresentação, exploramos uma série de hipóteses específicas com o objetivo de entender a transmissão intergeracional de status social e educacional. A perspectiva geral proposta aqui é que este fenômeno resulta de um processo de socialização que é simultaneamente cognitivo e social. Crianças de NSE baixo e médio constroem mundos consensuais diferentes que dão às crianças de classe média razões para aprender e às crianças de classe baixa razões para não aprender na escola. As diferenças que antecipamos encontrar não estão nas crenças gerais sobre o valor da educação, mas nas suas representações específicas de estratificação social e educacional, sua identidade neste mundo social e os tipos de conhecimento nos quais valeria a pena investir, considerando sua identidade. Antecipamos encontrar que crianças de NSE médio e baixo valorizem a educação e entendam que a educação traz maior status socioeconômico. No entanto, antecipamos que a própria história pessoal do NSE das crianças interaja com: (1) suas representações mais específicas de aspectos econômicos e educacional da estratificação social; (2) sua própria identidade dentro do sistema; e (3) sua visão do que o conhecimento pode fazer por eles. Nossa evidência provém, na sua maioria, de investigações realizadas no Brasil: nos referimos a resultados relevantes de outros países apenas brevemente para documentar que o fenômeno não é específico. A seguir examinaremos como crianças de diferentes NSE representam as conquistas sociais e educacionais.

\section{Representações sociais da estratificação: renda e níveis educacionais.}

Nossa primeira hipótese foi que crianças de diferentes NSE representam a estratificação social de maneiras que as motivam diferentemente para o aprendizado na escola.

Pesquisa sobre o entendimento de crianças sobre estratificação social em diferentes países mostra que crianças têm consciência de diferenças em salários e status social entre as profissões. Elas explicam estas diferenças atribuindo maior importância ao trabalho feito por pessoas em profissões com maiores salários e atribuem maiores níveis de competência aos profissionais mais bem pagos. Estes resultados foram observados tanto em crianças de NSE baixo como em crianças de NSE médio (Emler \& Dickinson, 1985; de Abreu, 1997; Jahoda, 1959, 1984). Assim, crianças de diferentes NSE compartilham a visão que a atribuição de status maior a dadas profissões é consequência da maior 
importância do trabalho feito e do maior nível de competência e educação daqueles que exercem a profissão.

No entanto, esta similaridade na visão de senso comum das consequências educacionais para a estratificação social não impede a existência de diferenças que possam resultar em motivações diferentes para ter êxito na escola.

Crianças de diferentes NSE têm níveis diferentes de diferenciação entre profissões nos grupos mais altos e mais baixos de estratificação social. Enquanto crianças de NSE baixo percebem distinções claras entre as profissões de menor salário e fazem menos distinções entre as profissões de salário menor, exatamente o oposto é observado em crianças de NSE médio e alto. Carraher e Carraher (1982) foram os primeiros a documentarem o alto nível de discriminação demonstrada por adolescentes pobres de ocupações de baixo status, perguntando a eles o que gostariam de se tornar em termos ocupacionais quando deixassem a escola, e por quê. Os adolescentes eram estudantes atendendo aulas noturnas para compensar um prévio atraso na sua progressão escolar. Eles surpreenderam os pesquisadores demonstrando uma representação altamente diferenciada de profissões de status mais baixo e considerando critérios de classificação que adolescentes de NSE médio não consideram. Por exemplo, ocupações que pareciam similares (e igualmente indesejáveis) a adolescentes de NSE médio foram diferenciados por aqueles de NSE baixo pelo fato de se o trabalho era feito na sombra ou no sol, sentado ou em pé, limpo ou sujo, sob pressão para o desempenho ou não etc. Ocupações como caixa num supermercado e telefonista foram considerados altamente desejáveis pelos adolescentes de NSE baixo: o trabalho é feito na sombra, sentado, é um trabalho limpo e a pressão para melhorar o desempenho é percebido como ausente. Carraher e Carraher sugeriram que a motivação destes alunos a progredirem na escola pode ser para atingir níveis educacionais compatíveis com as ocupações a que aspiravam, em vez de uma motivação geral a alcançar os níveis educacionais mais altos.

As recompensas de salário associadas com ocupações em diferentes posições na estratificação também são representadas diferentemente nos NSE diferentes. Crianças de NSE médio e baixo na França, nos Estados Unidos e na Escócia divergem em termos dos valores que atribuem aos salários nas profissões de status mais alto e mais baixo. Emler e Dickinson (1985) apresentaram uma lista de profissões a crianças de NSE diferentes e pediram que eles estimassem o valor dos salários para cada uma das profissões. Crianças de NSE baixo estimaram a diferença entre as ocupações mais e menos bem pagas sendo significativamente menor que a diferença entre salários estimada pelas crianças de NSE médio e alto.

Nós (Roazzi, Sales, Carvalho \& Nunes, 1997) duplicamos estes resultados no Brasil e os relacionamos às representações das crianças do nível de escolaridade necessário para as diferentes ocupações. Pediu-se a crianças ( $\mathrm{N}=180$, idades entre 7 e 12 anos) de diferentes NSE (baixo e médio) que estimassem os salários (pela distribuição de 80 fichinhas) e o nível educacional de 24 ocupações; metade das crianças de NSE baixo estavam frequentando a escola e metade tinham deixado a escola. As ocupações incluíam algumas que dependiam de formação universitária (e.g., médico), ocupações de alto status que não exigem formação universitário mas onde a educação é, de fato, necessária (e.g., gerente de banco, político), ocupações de colarinho branco de status inferior onde as exigências educacionais são indefinidas (e.g., policial), qualificações vocacionais (e.g., motorista de ônibus) e nenhuma qualificação exigida (e.g., gari). Antecipamos uma interação significativa entre NSE e as estimações das crianças de salário e qualificação educacional.

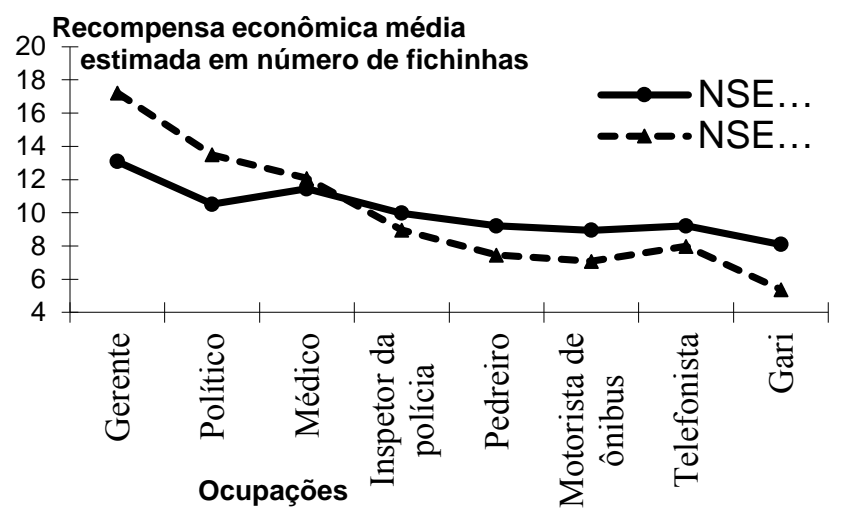

Figura 1.

Estimativa da recompensa relativa de diferentes ocupações por adolescentes de NSE baixo e médio

A Figura 1 mostra os salários médios em números de fichinhas atribuídos a uma amostra das ocupações por crianças de NSE baixo e médio. As ocupações aparecem na figura na ordem de acordo com o número médio de fichinhas atribuídos pela amostra total: representações de crianças atendendo e não atendendo a escola foram juntadas porque não houve diferenças entre os dois grupos. As estimativas de salários foram analisadas através de uma análise de variância tipo 2 (NSE) x 3 (níveis de idade) x 8 (profissões). Esta análise produziu interação significativa entre o status socioeconômico das crianças e o tipo de profissão, como antecipado $(F=6.786 ; p<.001)$ e os principais efeitos significativos de idade $(F=2.08 ; p<.012)$ e de profissão $(F=33.78$; $p<.001)$.

Pediu-se aos participantes também que indicassem quanta educação achavam que pessoas nestas ocupações precisariam para serem qualificados para seus empregos; respostas poderiam ser dadas em termos gerais (e.g., "formação universitária") ou termos específicos (e.g., "terminar até a oitava séria"). A Figura 2 mostra os níveis médios de educação estimada como necessária para as diferentes categorias educacionais; crianças dentro e fora da escola são consideradas separadamente por causa das diferenças entre suas representações. Níveis educacionais são medidos em anos e a estrutura do sistema escolar brasileiro é delineado no eixo. 


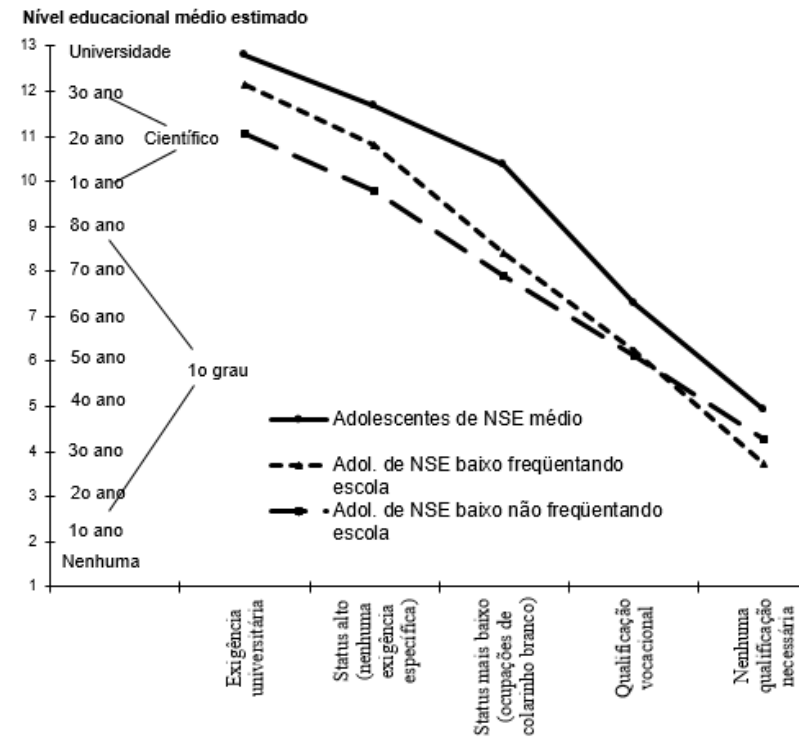

Figura 2. Estimativa dos níveis educacionais necessários para exercer diferentes ocupações de acordo com os adolescentes de NSE médio e baixo

Jovens de NSE baixo subestimaram o nível de educação necessário para algumas das ocupações mais prestigiosas, embora tenham sido bastante realistas em entender que as ocupações menos prestigiosas necessitam de pouca escolarização. As exigências educacionais para todas as ocupações foram estimadas em níveis mais altos pelas crianças de NSE médio. Uma análise de variância com Grupo (3: NSE alto, NSE baixo frequentando escola e NSE baixo não frequentando a escola) e Tipo de ocupação (5) como os fatores (entre e intra, respectivamente) e o julgamento do nível de educação como a variável dependente foi calculada para verificar se havia uma interação entre os NSE das crianças e a representação da relação entre sucesso educacional e ocupação. Esta análise produziu efeitos principais significativos para Grupo $(F=21.58 ; p<.001) \mathrm{e}$ Tipo de ocupação $(F=710.66 ; p<.001)$ e uma interação significativa $(F=4.85 ; p<.001)$. Comparações post-hoc de Tukey mostraram que adolescentes de NSE mais alto deram estimativas maiores para os níveis de educação necessários para as diferentes ocupações em comparação com ambos os grupos de adolescentes de NSE baixo; a única exceção foi a educação estimada para ocupações com o status mais baixo. Comparações entre os adolescentes de NSE baixo frequentando a escola e aqueles que tinham deixado a escola mostram que aqueles ainda na escola deram estimativas mais altas para os níveis de educação necessárias para as duas ocupações mais prestigiosas: outras diferenças em educação estimada não eram significativas quando estes dois grupos foram comparados. Para os três grupos de adolescentes, as diferenças educacionais percebidas foram significativas entre os diferentes tipos de ocupações. .

Em suma, estes estudos mostram similaridades e diferenças entre as representações da estratificação social e educacional de crianças de NSE médio e baixo. Ambos os grupos parecem reconhecer que qualificações educacionais estão associadas a status socioeconômico. No entanto, crianças de NSE baixo tendem a subestimar as exigências educacionais de ocupações de status mais alto e suas recompensas em comparação com ocupações de status mais baixo. Estas discrepâncias provavelmente resultam em diferenças na sua motivação de aprender na escola, particularmente numa sociedade onde a educação é vista como um meio para o sucesso social e econômico.

\section{Aprender e tornar-se alguém: identidade ocupacional e desenvolvimento na escola.}

Na seção anterior foram apresentadas evidências sobre como crianças de diferentes NSE representam conquistas sociais e educacionais. Na apresentação que será exposta no simpósio examinaremos em seguida como as crianças aplicam estas categorias para si mesmas. Esta parte não consta neste trabalho em decorrência de limites de páginas dos Anais.

\section{A marcação social de estruturas de conhecimento}

Nossa terceira hipótese é que o conhecimento é socialmente marcado e que o conhecimento que adolescente aspira a adquirir é relacionado com sua identidade. A primeira indicação de marcação social do conhecimento que observamos no Brasil foi relacionado ao uso dos termos "alfabetizado" e "analfabeto": por exemplo, o mesmo professor universitário no Brasil poderá dizer que sua cozinheira, a qual deixou a escola depois da segunda série, é "alfabetizada" mas que seus alunos universitários são "analfabetos" (Nunes Carraher, 1986; 1987). O que é implícito nestas suposições é que a cozinheira é alfabetizada para seus propósitos enquanto os alunos universitários não estão no nível esperado de alfabetização: "alfabetização", portanto, não é uma competência cognitiva neutra, mas uma forma de conhecimento socialmente marcado.

A marcação social do conhecimento no Brasil foi também documentada no âmbito do conhecimento matemático. Nunes, Schliemann e Carraher (1993) observaram que jovens vendedores de rua eram bastante capazes de resolver problemas aritméticos quando vendendo produtos nos mercados de rua (um média de sucesso de 98\%). No entanto, quando pediu-se que resolvessem somas numa situação similar a um teste, em vez de usar métodos de cálculo orais, que usam com tanto sucesso quando calculando no mercado de rua, na maioria das vezes os jovens escolheram usar algoritmos escritos, que não dominavam, e muitas vezes levavam ao erro (uma média de sucesso de apenas 37\%). Uma discrepância similar entre competência oral e escrita na resolução de problemas foi observada posteriormente por Nunes (1993) no âmbito de números negativos: jovens frequentando aulas noturnas ( $\mathrm{da} 4^{\mathrm{a}}$ à $6^{\mathrm{a}}$ série) tinham bem mais probabilidade de ter sucesso em resolver problemas com números negativos se usassem cálculo oral em vez de escrita, e também tinham consciência da sua maior habilidade em cálculo oral. Esta discrepância em desempenho por jovens pobres em aritmética oral versus escrita apresentou um enigma: por que as crianças 
escolhiam resolver os itens de teste usando aritmética escrita quando eram melhores com a aritmética oral e reconheciam esta diferença na sua habilidade?

De Abreu (1997) estudou esta questão num estudo posterior e criou a hipótese de que a explicação estaria na marcação social do conhecimento: professores e alunos na escola primária atribuem valores diferentes a estas duas formas de prática numérica e que esta diferença de valor percebido afeta as escolhas dos alunos de que forma de conhecimento usar em diferentes situações, como também que forma de conhecimento aspiram a aprender. De Abreu testou esta hipótese num estudo de uma comunidade rural pobre no nordeste do Brasil, onde a maioria dos trabalhadores em agricultura sem escolarização teriam sido expostos a práticas de aritmética oral.

De Abreu entrevistou crianças na escola, seus professores, e seus pais sobre sua consciência da existência de práticas aritméticas diferentes e sua valorização destas práticas. Ela descobriu que tanto os professores como os alunos consideravam a aritmética oral usada fora da escola como "prática" e a aritmética escolar como "teórica". Muitas vezes reconheceram que ambas as formas de conhecimento matemático podem levar a resultados corretos; no entanto, valorizavam as práticas diferentemente.

Os professores tinham consciência de que a matemática da rua poderia ser eficaz e mesmo impressionante (um professor admitiu que seu pai sem escolarização podia calcular melhor usando a aritmética oral do que ela com seus métodos escolares). Mas na escola, ela procurava ensinar apenas os algoritmos escritos, que eram percebidos como o meio ao sucesso no sistema educacional.

As atitudes dos alunos variavam: alguns alunos consideravam que a aritmética oral leva a resultados corretos mas não achavam que era "a maneira correta" de fazer somas, enquanto outros valorizavam-na como altamente eficaz. A mesma diversidade foi observada com respeito aos métodos escolares: alguns alunos consideravam-nos menos eficazes que a aritmética oral, enquanto outros consideravam-nos importante junto com ou em oposição a métodos orais.

De Abreu trabalho ou alunos mais novos ( $3^{\mathrm{a}}$ série) e mais velhos ( $5^{\mathrm{a}}$ série). Entre os alunos mais velhos, em particular, o sucesso nos métodos escolares foi associado a uma atitude positiva para com eles, com ou sem a desvalorização da aritmética oral. Seu desempenho em aritmética oral, no entanto, era claramente independente do seu desempenho na matemática escolar: alunos que tinham sucesso nos métodos escolares não tinham mais probabilidade nem de conhecer nem de não conhecer as práticas de aritmética oral usadas fora da escola. De Abreu ressalta que à medida que os alunos envelhecem, alguns ficam mais envolvidos em atividades produtivas fora da escola. Para estes alunos, a identificação com a escola como um lugar de aprendizado diminui: eles valorizam o trabalho que fazem do lado de fora e a matemática que aprendem na prática: eles vêm a desvalorizar a matemática escolar como "teórica demais" e pouco prática para a resolução de problemas na vida diária. Estes alunos têm bem mais probabilidade do que outros a mostrar uma discrepância marcante entre seu conhecimento de práticas orais e escolares escritas.

Em suma, De Abreu não encontrou relação entre os dois tipos de conhecimento ao nível cognitivo mas encontrou que alunos que valorizavam a matemática escolar eram mais prováveis a ter sucesso nesta forma de matemática.

Estudos em outros países também indicaram os conflitos entre a identidade que o indivíduo poderia ter tornando-se uma pessoa escolarizada e a identidade presente dos adolescentes de grupos minoritários ou de NSE baixo. Willis (1977) documentou este conflito em garotos de classe trabalhadora na Inglaterra, Ahai e Faraclas (1993) entre adolescentes que denomina patifes ("rascals") em Papua Nova Guiné, e Ogbu entre negros norte-americanos; Singh também indica os mesmos conflitos para crianças Ho na Índia. Em todos estes exemplos, as conexões entre quem os alunos são, como percebem o conhecimento escolar, que tipo de conhecimento valorizam e quem aspiram a se tornar são intimamente relacionados com as razões dos alunos para aprender ou não na escola.

\section{Conclusões}

A evidência aqui considerada dá apoio à teoria de que, embora jovens de diferentes NSE valorizam a educação e reconhecem seu papel na estratificação socioeconômica, eles representam as especificidades da estratificação social diferentemente.

Para jovens de NSE médio, as ocupações de status mais alto estão diferenciadas de acordo com suas preferências: podem querer ser médicos, engenheiros ou advogados dependendo de objetivos pessoais. Mas não há discrepância entre o que idealmente gostariam de tornar-se e o que acreditam que seja provável que se tornem. As ocupações de status mais baixo são todas igualmente indesejáveis e não precisam ser distinguidas. Exigências educacionais para todas as ocupações são estimadas a valores mais altos que as estimativas dos jovens de NSE baixo; as recompensas por um nível mais alto de educação também são estimadas num nível mais alto.

Em contraste, jovens de NSE baixo, em comparação, subestimam níveis de educação necessários para ocupações, como também as recompensas por níveis mais altos de escolarização. Eles preveem uma diferença significativa entre o que idealmente gostariam de tornar-se e o que provavelmente se tornarão. Como consequência, fazem distinções mais detalhadas entre as ocupações de status mais baixo e expressam a intenção de evitar as ocupações indesejáveis quando possível. Também consideram algum do conhecimento que são ensinados na escola como distantes das suas necessidades práticas: eles aprendem a valorizar conhecimento que não é ensinado na escola mas é útil para os tipos de atividades que de fato vão exercer.

Em conclusão, ressaltamos a necessidade de mais pesquisa sobre este tema, particularmente para analisar as interações entre desempenho escolar e possíveis mudanças ou fortificação de identidades específicas ao longo do tempo. No entanto, vale a pena considerar, 
mesmo que brevemente, os desafios provindos desta análise para a prática educacional: é possível mudar a marcação social do conhecimento conferindo reconhecimento oficial no currículo escolar ao conhecimento prático ou do dia-a-dia. Como podem as escolas estarem mais abertas a alunos de todos os NSE para fazer o aprendizado compatível com uma variedade de identidades?

\section{Referências}

Ahai, N. and N. Faraclas (1993). Rights and expectations in and age of "Debt Crisis": Literacy and integral human development in Papua New Guinea. Em P. Freebody \& A. R. Welch (Eds.), Knowledge, culture, and power: International perspectives on literacy as policy and practice (pp. 82-101). London: Falmer Press.

Aitkin, M., Anderson, D., Francis, B. \& Hinde, J. (1989). Statistical modelling in GLIM. Oxford: Clarendon Press.

Baker, R.J. \& Nelder, J.A. (1978). The Glim System Release 3 Manual. Oxford: Numerical Algorithms Group and Royal Statistical Society.

Bourdieu, P. \& J. C. Passeron (1977). Reproduction in education, society and culture. London: Sage.

Bourdieu, P. (1974). The school as a conservative force; scholastic and cultural inequalities. Em J. Eggleston (Ed.), Contemporary research in the sociology of education (pp. 32-46). London: Methuen.

Carraher, D. W. \& T. N. Carraher (1982). O que eu mais gostaria de ser é caixa num supermercado. O dilema das aspirações ideais deprimidas. Sociedade Brasileira para o Progresso da Ciência. Reunião Anual. Rio de Janeiro: Brasil.

De Abreu, G., Pompeu, G, \& Bishop, A. (1997). What children and teachers count as mathematics. Em T. Nunes \& P. Bryant (Eds.), Teaching and learning mathematics. An international perspective. New York: Lawrence Erlbaum Associates.

Emler, N. \& Dickinson, J. (1985). Children's representations of economic inequality. British Journal of Developmental Psychology, 3, 191-198.

French, J. (1990). Social interaction in the classroom. Em C. Rogers \& P. Kutnick (Eds.), The social psychology of the primary school (pp. 31-48). London: Routledge.

Healy, M.J.R. (1988). Glim: An introduction. Oxford: Clarendon Press.

Herrnstein, R. (1971). IQ. The Atlantic, (September), 43-64.

Jahoda, G. (1959). Development of the perception of social differences in children from six to ten. British Journal of Psychology, 50, 158-196.

Jahoda, G. (1984). The development of thinking about socio-economic systems. Em H. Tajfel (Ed.), The social dimension: European developments in social psychology. Cambridge: Cambridge University Press.

Jensen (1969). How much can we boost IQ and scholastic achievement? Harvard Educational Review, 39, 1-123.

Nunes Carraher, T. (1986). Alfabetização e pobreza: Três faces do problema. Em S. Kramer (Ed.), Alfabetização. Dilemas da prática (pp. 47-98). Rio de
Janeiro: Dois Pontos.

Nunes Carraher, T. (1987). Illiteracy in a literate society. In D. Wagner (Ed.), The future of literacy in a changing world (pp. 95-110). Oxford: Pergamon Press.

Nunes, T. (1993). Learning mathematics: Perspectives from everyday life. Em R. B. Davis \& C. A. Maher (Eds.), Schools, mathematics, and the worlds of reality (pp. 61-78). Needham Heights (MA): Allyn and Bacon.

Nunes, T., Schliemann, A. D., \& Carraher, D. W. (1993). Street mathematics, school mathematics. Cambridge: Cambridge University Press.

Ogbu, J. (1983). Literacy and schooling in subordinate cultures: The case of black Americans. Em D. P. Resnick (Ed.), Literacy in historical perspective (pp. 117-138). Washington, DC: Library of Congress.

Roazzi, A., Sales, L.C., Carvalho, \& Nunes, T. (1997). A representação de status social entre adolescentes de diversos meios sociais. Recife (PE): Brasil, Universidade Federal de Pernambuco.

Terman, L. M. \& Merril, M. A. (1937). Measuring intelligence. Boston: Houghton Mifflin.

Willis, P. (1977). Learning to labour. How working class kids get working class jobs. London: Saxon House. 